\title{
Thermally stimulated discharge conductivity study of zinc oxide thermoelectrets
}

\author{
VIJAYA S SANGAWAR and MANISHA C GOLCHHA* \\ Polymer/Nanomaterials Research Laboratory, Govt. Vidarbha Institute of Science and Humanities, \\ Amravati 444 602, India
}

MS received 11 November 2013; revised 27 December 2013

\begin{abstract}
The present work deals with transmission electron microscopy (TEM), X-ray diffraction (XRD), differential scanning calorimetry (DSC) and thermally stimulated discharge current (TSDC) study of inorganic metal oxide ( $\mathrm{ZnO})$ nanoparticles and its thermoelectrets. The thermoelectrets were prepared by applying different electric polarizing field $\left(E_{\mathrm{P}}\right)$ at constant polarizing temperature $\left(T_{\mathrm{P}}\right)$, for constant polarization time $\left(t_{\mathrm{p}}\right)$. The TSDC study was carried out in the temperature region of 313-473 K. It was observed that the conductivity of $\mathrm{ZnO}$ samples increases with the increase in temperature and polarizing field. The dependence of TSDC data on polarizing agents, i.e. field and temperature shows Arrhenius type of behviour and is explained on the basis of variable range hopping mechanism.
\end{abstract}

Keywords. ZnO nanoparticles; DSC; thermoelectrets; TSDC.

\section{Introduction}

As we all know, any material in its nano form is more demandable than in its bulk form, because at nano level, the material undergoes a drastic change in its properties and has versatile applications. Recently, nanoparticles (NPs) of metal oxides have been the focus of a number of research efforts due to the unusual physical properties that are expected on entering this size regime. Control and manipulation of the morphology of the NPs will ultimately dictate the electrical and optical properties of the final devices (Wu et al 2006).

Zinc oxide $(\mathrm{ZnO})$ is a semiconductor compound characterized by wide band gap (3.37 eV) and a larger excition binding energy (60 meV). Moreover, $\mathrm{ZnO}$ is very important material because of its interesting properties, such as dielectric, piezo-electric, pyro-electric, semiconducting, acousto-optic, optical, electro-optical, nonlinear optical, photo-electrochemical and electrical properties (Chu et al 2000). These properties make $\mathrm{ZnO}$, a promising material to be used in ultraviolet light-emitting diodes, photodetectors, solar cells and surface acoustic wave devices (Salama and Hammad 2009). The applications of $\mathrm{ZnO}$ for the above devices are associated with the transport mechanism of charge carriers in this material. For the effective functioning of these devices, the study and knowledge of transport mechanism is very important.

\footnotetext{
*Author for correspondence (manisha_golchha22@yahoo.co.in)
}

An electret is a piece of dielectric material that is capable of storing charges in a quasi-permanent state and finds an important place in several areas including signal transmission and reception. The ZnO NPs electrets were prepared by thermal polarization method. The thermally stimulated discharge current (TSDC) technique is much less employed to characterize electrical behaviour of inorganic dielectric materials. However, it is a powerful tool in the study and quantification of defects, and more important to study the transport of charge carriers, i.e. oxygen vacancies. This paper reports the study of temperature dependence of conductivity of $\mathrm{ZnO}$ NPs along with the TSDC study. The conduction mechanism in ZnO NPs is found to be via activated type and variable range hopping conduction processes.

\section{Materials and method}

The hexagonal wurtzite structured ZnO NPs with average crystallite size about $80 \mathrm{~nm}$ were successfully synthesized by simple chemical solution method followed by combustion. Detailed preparation technique and properties of as-prepared NPs was reported elsewhere (Golchha et al 2011). The morphology of NPs was observed with a TEM, Hitachi H-7100 transmission electron microscopy. $\mathrm{X}$-ray diffraction (XRD) data of the as-synthesized $\mathrm{ZnO}$ NPs was collected using Phillips Holland, XRD system PW 1710 diffractometer using $\mathrm{CuK} \alpha$ radiation ( $\lambda=$ $0 \cdot 154056 \mathrm{~nm}$ ). Differential scanning calorimetry (DSC) was used to obtain the thermal properties of oven-dried 
ZnO NPs. DSC measurements were carried out using a Netzsch DSC 200F3 PC instrument in the temperature region from room temperature to $450{ }^{\circ} \mathrm{C}$, with heating rate of $10^{\circ} \mathrm{C} / \mathrm{min}$ in nitrogen atmosphere.

In order to measure the electrical properties, $\mathrm{ZnO} \mathrm{NPs}$ were grounded into fine powder and the pellets of diameter $1.16 \mathrm{~cm}$ were fabricated using a manual hydraulic press machine. The pellet was then annealed at $500{ }^{\circ} \mathrm{C}$ for $1 \mathrm{~h}$ before current measurements. The thickness of the sample was measured by the compound microscope in conjunction with an occulometer having least count of $15.38 \mu \mathrm{m}$, similar to method reported by Sangawar et al (2006). The thickness of the sample was kept constant throughout the work and was of the order of $0.1692 \mathrm{~cm}$.

Before TSDC measurement, polarization of sample was carried out by first heating them to a specific polarizing temperature, $T_{\mathrm{p}}=353 \mathrm{~K}$ and then subjecting the sample to different electric polarizing fields, $E_{\mathrm{p}}(0,1,2$ and $3 \mathrm{kV} / \mathrm{cm}$ ) using a stabilized 'Labin' d.c. voltage source, for a constant polarizing time, $t_{\mathrm{p}}$. After polarization, the sample was cooled to room temperature under continuing electric stress. Total polarization time, $t_{\mathrm{p}}$, was adjusted to $2 \mathrm{~h}$ in each case. The sample was then short-circuited for $20 \mathrm{~min}$ to remove frictional and stray charges, if any.

After the electret formation, the sample was placed in the sample holder of brass electrodes (M-S-M sandwich configuration) and then placed in a controlled temperature furnace for TSDC study. The sample was thermally discharged in the temperature region of 313 $473 \mathrm{~K}$ at a uniform rate of $2{ }^{\circ} \mathrm{C} / \mathrm{min}$ and the corresponding current was measured across the sample using a Digital Nanometer DPM III, supplied by Scientific Equipments, Roorkee. The method for conductivity measurement was the same as that reported earlier (Sangawar and Moharil 2012).

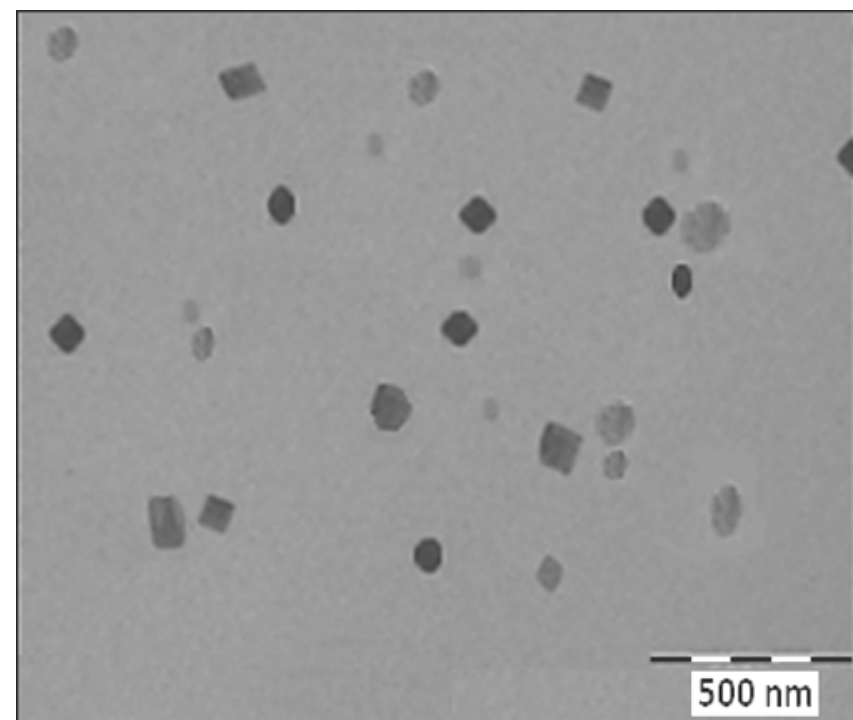

Figure 1. TEM image of $\mathrm{ZnO}$ nanoparticles.

\section{Results and discussion}

\subsection{Tunneling electron microscopy (TEM) analysis}

Morphology of synthesized ZnO NPs observed using TEM image is presented in figure 1 . It shows that the synthesized ZnO NPs have spherical morphology with some tetragonal NPs.

\subsection{XRD analysis}

Figure 2 shows the XRD pattern of the as-prepared $\mathrm{ZnO}$ NPs. The diffraction peaks appeared at $2 \theta=31.70^{\circ}$, $34.40^{\circ}, 36 \cdot 20^{\circ}, 47.50^{\circ}$ and $56 \cdot 60^{\circ}$, which corresponds to ( $\left.\begin{array}{lll}1 & 0 & 0\end{array}\right),\left(\begin{array}{lll}0 & 0 & 2\end{array}\right),\left(\begin{array}{lll}1 & 0 & 1\end{array}\right),\left(\begin{array}{lll}1 & 0 & 2\end{array}\right)$ and $\left(\begin{array}{lll}1 & 1 & 0\end{array}\right)$ planes in hexagonal wurtzite structure consistent with the bulk ZnO (JCPDS card no. 36-1451) (Cheng-Hsien 2007; Nirmala et al 2010). As shown in the XRD data, the ( $\left.\begin{array}{lll}1 & 0 & 0\end{array}\right)$ direction in figure 2 is the preferred orientation for the synthesized ZnO NPs. The strong and sharp peaks reveal the highly crystallite nature of NPs. The average crystallite size was found to be about $80 \mathrm{~nm}$, calculated using the Scherrer formula

$$
D=\frac{0 \cdot 9 \lambda}{\beta \cos \theta}
$$

where $D$ is the crystallite size, $\lambda$ the wavelength of X-ray, $\beta$ the FWHM in radian and $\theta$ the peak angle.

\subsection{DSC analysis}

A DSC thermogram is shown in figure 3. It reveals endothermic peaks when the decomposition occurs. The curve shows small endothermic peaks around 143 and $171.5{ }^{\circ} \mathrm{C}$. These peaks are attributed to the evaporation of water and

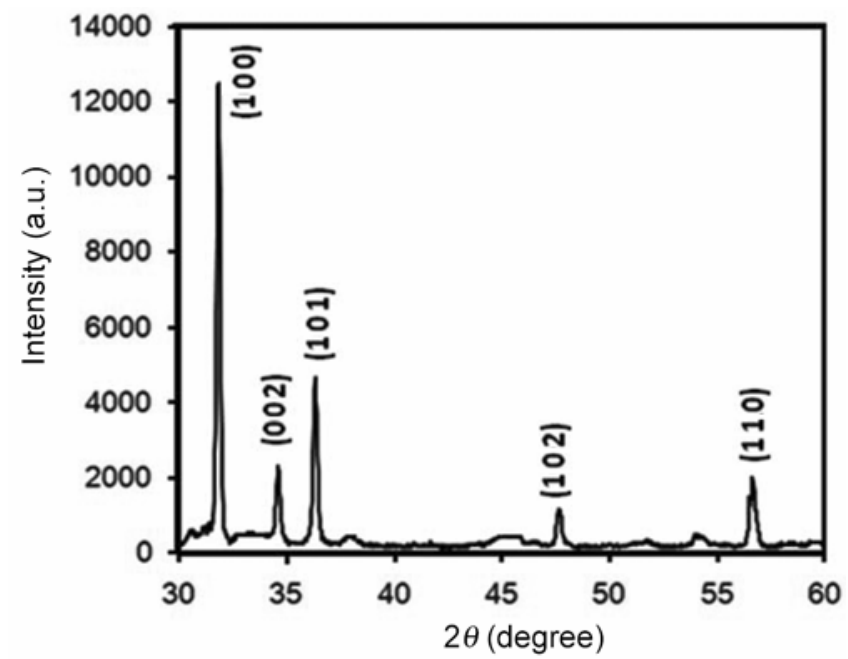

Figure 2. XRD pattern of $\mathrm{ZnO}$ nanoparticles. 


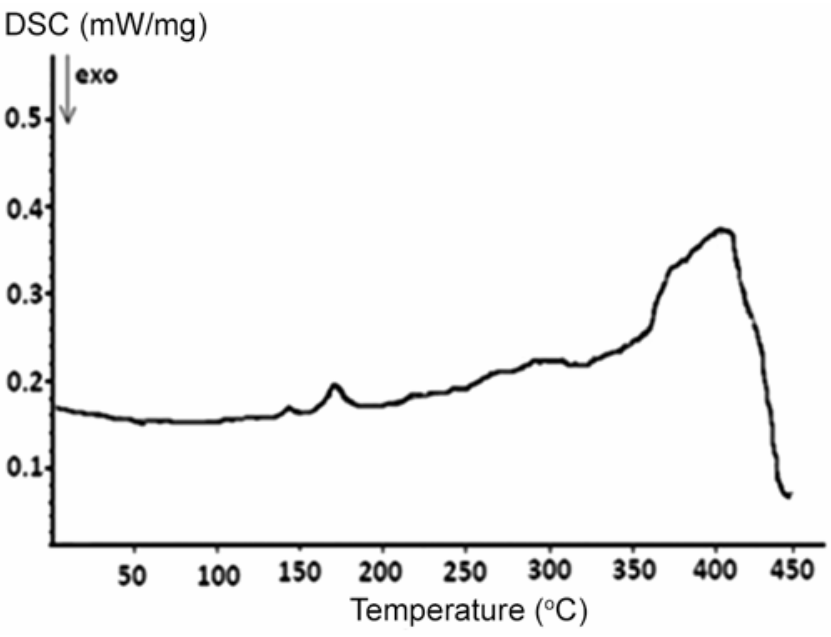

Figure 3. DSC curve of $\mathrm{ZnO}$ nanoparticles.

decomposition of organics. While endothermic peaks $403.4{ }^{\circ} \mathrm{C}$ probably represents the removal of oxalate species (Chen et al 2008; Pillai et al 2008; Telipan et al 2011).

\subsection{D.C. electrical conductivity}

Thermal treatment to the respective sample modifies their structural characteristics and consequently, their electrical properties. The temperature dependence of d.c. electrical conductivity $\left(\sigma_{\text {d.c. }}\right)$ of $\mathrm{ZnO}$ nano-powder pelleted samples was obtained by measuring the current across the sample as a function of temperature (in the range of 313-473 K) at different poling fields, i.e. $0,1,2$ and $3 \mathrm{kV} / \mathrm{cm}$. Figure 4 shows the thermograms ( $\log \sigma$ vs $10^{3} / T$ plot) of the $\mathrm{ZnO}$ samples.

The values of electrical conductivities have been obtained using the relation

$$
\sigma=[(I \times t) /(V \times A)],
$$

where $I$ is the current, $V$ the voltage, $t$ the thickness and $A$ the cross-sectional area.

From figure 4, it is observed that the d.c. electrical conductivity of polarized $\mathrm{ZnO}$ samples (thermoelectrets) is higher than the unpolarized $\mathrm{ZnO}$ sample. For all the samples, conductivity increases with polarizing field.

It is also observed that the conductivity increase with increase in temperature, represents semiconducting nature. It can be noticed that the conductivity increases with the temperature obeying the well-known Arrhenius equation

$$
\sigma=\sigma_{0} \exp \left[\left(-\frac{E_{\mathrm{a}}}{k T}\right)\right],
$$

where $\sigma$ is the electrical conductivity at any temperature, $\sigma_{0}$ the pre-exponential factor, $E_{\mathrm{a}}$ the activation energy,

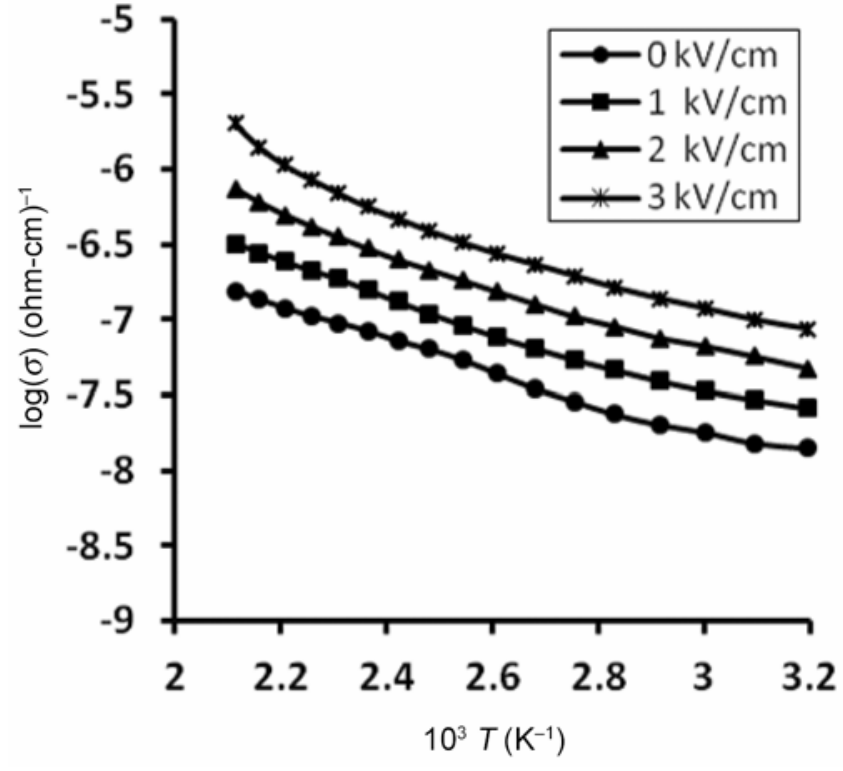

Figure 4. Plots of $\log \sigma$ vs $1 / T \times 10^{3}$.

Table 1. Activation energy values.

\begin{tabular}{lcc}
\hline & \multicolumn{2}{c}{$E_{\text {a values }}(\mathrm{eV})$} \\
\cline { 2 - 3 } Field $(\mathrm{kV} / \mathrm{cm})$ & LTR & HTR \\
\hline 0 & 0.144 & 0.205 \\
1 & 0.147 & 0.232 \\
2 & 0.150 & 0.310 \\
3 & 0.161 & 0.409 \\
\hline
\end{tabular}

which corresponds to the energy difference between the donor level and the conduction level, $k$ the Boltzmann constant and $T$ the absolute temperature (Hossain et al 2008; Natsume et al 2000).

The activation energy values in low temperature region (LTR) and high temperature region (HTR) have been calculated from the slope of thermograms and are shown in table 1. $E_{\mathrm{a}}$ values for all the samples are found to be lower in LTR as compared to HTR. In both the temperature regions, $E_{\mathrm{a}}$ values are found to be increased with increase in polarizing field and temperature. This can be due to the energy gap width at low temperature region is lower than that of the high temperature region, which was attributed to the increased ionized oxygen vacancy $V_{0}$ with the increase in temperature (Salama and Hammad 2009).

At low temperature, when the mobility of the charge carriers is low, the charges may be largely localized in shallow traps. However, at high temperature, the charge carriers with high mobility are shifted to deeper traps. The number of such trapped charge carriers increases with the polarizing field. Thus, as the temperature increases, mobility of the charge carriers also increases; consequently shifting the excess charges to deeper traps. 


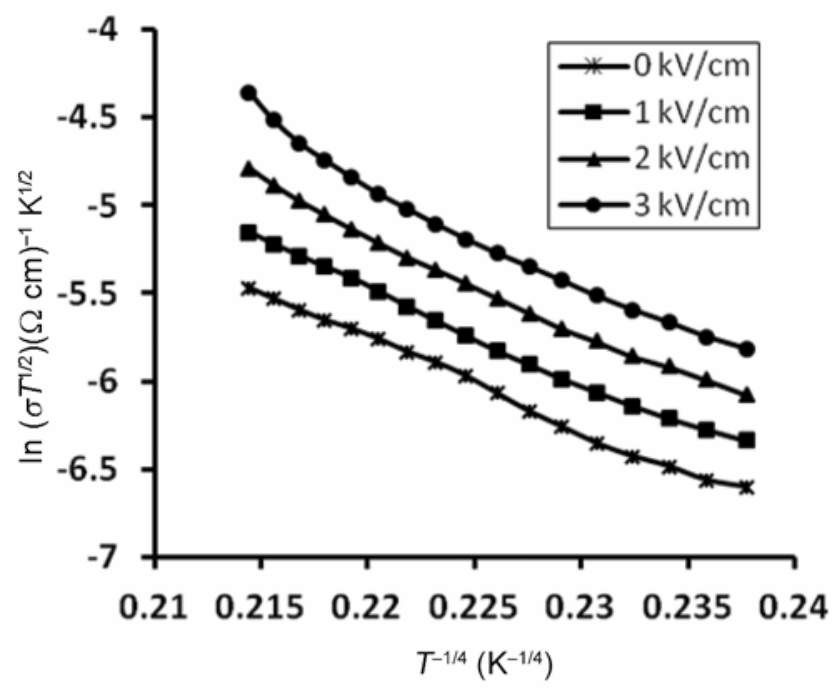

Figure 5. Plot of $\ln \left(\sigma T^{1 / 2}\right)$ vs $T^{-1 / 4}$.

The release of charge carriers from such traps requires more energy.

Variable range hopping (VRH) is also considered as one of the major carrier-transport mechanism proposed for inorganic semiconductors. Hopping refers to tunnelling transition from occupied to unoccupied localized status. In the VRH conduction mechanism, the electrons hop between the levels that are close to Fermi level irrespective of their spatial distribution. So in this type of hopping conduction, the hopping distance is not constant as in the nearest-neighbour hopping. The relation of conductivity with temperature for VRH is given by (Mott and Davis 1979)

$$
\sigma=\sigma_{\mathrm{h} 0} \exp \left[\left(-\frac{T_{0}}{T}\right)\right]^{1 / 4},
$$

where $\sigma_{\mathrm{h} 0}$ and $T_{0}$ are given by the following expressions

$$
\begin{aligned}
& \sigma_{\mathrm{h} 0}=\frac{3 e^{2} v_{\mathrm{ph}}}{(8 \pi)^{1 / 2}}\left[\frac{N\left(E_{\mathrm{F}}\right)}{\alpha k T}\right]^{1 / 2}, \\
& T_{0}=\left[\frac{16 \alpha^{3}}{k N\left(E_{\mathrm{F}}\right)}\right],
\end{aligned}
$$

where $v_{\mathrm{ph}}\left(\approx 10^{13} \mathrm{~s}^{-1}\right)$ is the phonon frequency at Debye temperature, $k$ Boltzmann's constant, $N\left(E_{\mathrm{F}}\right)$ the density of localized electron states at Fermi level and $\alpha$ the inverse localization length of the localized state.

From (5) and (6), we have

$$
\ln \left(\sigma T^{1 / 2}\right) \propto T^{-1 / 4} .
$$

Figure 5 represents the plot of $\ln \left(\sigma T^{1 / 2}\right)$ vs $T^{-1 / 4}$ for $\mathrm{ZnO}$ thermoelectrets indicating that the dominant mechanism of conduction is variable range hopping.
$\mathrm{ZnO}$ crystals exhibit strong $n$-type conductivity due to intrinsic defects (oxygen vacancy and interstitial zinc atoms) (Zhou et al 2004; Pearton et al 2005). These defects introduce donor states in the forbidden band slightly below the conduction band and hence, result in the conducting behaviour of $\mathrm{ZnO}$. The electrical conductivity is thus controlled by the intrinsic defects generated during synthesis (Sawalha et al 2009).

Moreover, $\mathrm{ZnO}$ is piezoelectric material and the origin of the piezoelectricity lies in its crystal structure, in which the oxygen and zinc atoms have tetrahedral bonding. In such a non-centrosymmetric structure, the centre of positive and negative charges can be displaced due to external pressure-induced lattice distortion. This displacement results in local dipole moment, thus, a macroscopic dipole moment appears over the whole crystal (Wang 2004).

Thus, while polarizing the ZnO NPs samples with polarizing electric field, $E_{\mathrm{p}}$, at an elevated temperature, $T_{\mathrm{p}}$, the possible defects that exist in the system respond to this field stress to form a meta-stable state of the charge or dipole distribution. Whereas, cooling the sample to a lower temperature $T_{0}$, freeze in those polarized defects. In TSDC process, the electric field was removed, and the sample is thermally discharged at a constant heating rate. As the temperature increases, the thermal energy excites the lattice vibrations, activates charge motion or aligned dipoles, resulting in the relaxations of those charge distributions or polarized states, and gives rise to a TSD current in the external circuit.

\section{Conclusions}

Differential scanning calorimetry (DSC) revealed that ZnO NPs were thermally stable. The d.c. electrical conductivity of ZnO NPs and its thermoelectrets in the temperature range from 313 to $473 \mathrm{~K}$, found to be increased with increasing temperature as well as polarizing field. The activated type of conduction as well as Mott's variable range hopping conduction mechanisms were found to be dominating. In addition, the activation energy of $\mathrm{ZnO}$ thermoelectrets were found to be increased with increasing temperature as well as polarizing field.

\section{References}

Chen K J, Fang T H, Hung F Y, Ji LW, Chang S J, Young S J and Hsiao Y J 2008 Appl. Surf. Sci. 2545791

Cheng-Hsien H 2007 J. Chin. Chem. Soc. 5431

Chu S Y, Yan T M and Chen S L 2000 Ceram. Int. 26733

Golchha M C, Bhagat R N and Sangawar V S 2011 Int. J. Nanotechnol. Appl. 5109

Hossain M S, Islam R and Khan K A 2008 Chalcogenide Lett. 51 Mott N F and Davis E A 1979 Electronic processes in noncrystalline materials (London: Oxford University Press) 
Natsume Y and Sakata H 2000 Thin Solid Films 37230

Nirmala M, Nair M G, Rekha K, Anukaliani A, Samdarshi S K and Nair R G 2010 Afr. J. Basic Appl. Sci. 2161

Pearton S J, Norton D P, Ip K and Heo Y W 2005 Prog. Mater. Sci. 50293

Pillai S C, Kelly J M, McCormack D E and Ramesh R 2008 J. Mater. Chem. 183926

Salama A H and Hammad F F 2009 J. Mater. Sci. Technol. 25314

Sangawar V S and Moharil N A 2012 Chem. Sci. Trans. 1447
Sangawar V S, Chikhalikar P S, Dhokne R J, Ubale A U and Meshram S D 2006 Bull. Mater. Sci. 29413

Sawalha A, Abu-Abdeen M and Sedky A 2009 Physica B 404 1316

Telipan G, Pislaru-Danescu L, Marinescu V, Prioteasa P and Zarnescu G 2011 Optoelectron. Adv. Mater. - Rapid Commun. 5643

Wang Z L 2004 J. Phys.: Condens. Matter 16 R829

Wu L, Wu Y and Lu Y 2006 Mater. Res. Bull. 41128

Zhou Z, Kato K, Komaki T, Yoshino M, Yukawa H, Morinaga M and Morita K 2004 J. Eur. Ceram. Soc. 24139 